\title{
Errors and Power When Communicating With Spins
}

\author{
EROL GELENBE, (Fellow, IEEE) \\ Intelligent Systems and Networks Group, Department of Electrical and Electronic Engineering, Imperial College London, \\ London SW7 2AZ, U.K. (e.gelenbe @imperial.ac.uk) \\ This work was supported by the U.K. Engineering and Physical Science Research Council through the \\ European Research Area Networks Energy harvesting Communication netwoRks: OPtimization and demonStration \\ Project under Grant EP/K017330/1.
}

\begin{abstract}
We consider a network composed of a finite set of communicating nodes that send individual particles to each other, and each particle can carry binary information. Though our main motivation is related to communications in nanonetworks with electrons that carry magnetic spin as the bipolar information, one can also imagine that the particles may be molecules that use chirality to convey information. Since it is difficult for a particle to carry an identifier that conveys the identity of the source or destination, each node receives particles whose source cannot be ascertained since physical imperfections may result in particles being directed to the wrong destination in a manner that interferes with the correctly directed particles, and particles that should arrive at a node may be received by some other node. In addition, noise may randomly switch the polarity of particles, and in the case of magnetic spin, we can also have the effect of entanglement. We estimate the error probability in such a multipoint network as a function of the rate of flow of particles, and the power consumption per communicating pair of nodes. We then design a bipolar detector and show that it can significantly eliminate the effect of errors.
\end{abstract}

INDEX TERMS Electron spin, nanonetworks, energy, interference, error probabilities, bipolar detector, quantum entanglement.

\section{INTRODUCTION}

Computing devices are composed of modules which carry out computational steps, whose results need to be communicated as inputs to other modules. Thus "on device" computation and communication are intricately related. The massive and increasing energy use by information and telecommunication technology reaching $4.7 \%$ of the world's electricity consumption [19], [20] motivates significant interest in low power computing communications including nano-networks at the molecular scale [27]. In addition to conventional techniques based on smart management of existing technologies [24], computation and communication with spins [5], [6], [26] may in the long run find useful low power applications. In any computation or communication system, energy consumed per computational step, and performance including the probability of error which ultimately affects response times, are intimately related [23], and many recent applicatons based on massive simulations [21] require intense communications and computation. Thus one must consider technologies that are require little energy, and yet result in low error probabilities [8], [13] since errors will have to be mitigated by hardware redundancy, temporal redundancy such as retransmissions, or the reprocessing of data, resulting in additional overhead and additional energy consumption.

In this paper we consider a network of nodes that communicate using flows of particles that carry simple bipolar information: we can think of the particles as electrons, and we can think of bipolar information as being spins [12].

In the model we consider, the received spins can be corrupted by three effects: the random switching of polarity or spin noise [17], the routing of some of the flows to the wrong receiving node (e.g. due to physical imperfections in the network) causing interference among distinct streams, quantum entanglement between particles' spin, and finally the injection of noise at the receivers. Since there is no way to "number or individually identify" the successive particles in a way that weeds out the ones arriving "accidentally" from the wrong source, or of maintaining all the correctly arriving particles in sequence [10], the interference we mention takes the form of misdirected particles arriving to a node, which may carry a spin polarity different from the intended one. 
Obviously the particles' spin must be detected at the receivers [11], [16] and the difference between the original intentionally imparted spin and the spin of the particles received at the receiver results in probabilistic errors. Assuming Poisson flows of particles emanating from the sources, we compute in Section III the error probability per particle received for a detector that recognises the polarity of the particles at the receiver using methods usually asociated with network performance analysis [1], [2], [4], [9], [22]. The energy consumption per correctly received particle is also discussed.

Detection of spins [16] can be achieved with ferromagnetic devices. Thus in Section IV we suggest a detector based on two bipolar counters or detectors: one of them increments its state with arriving positive spins and decrements it with negative spins, and the other has the opposite behaviour. Both counters have an identical random decay which limits their memory span. We show that if the incoming flow of particles is split equally at the receiver between the two counters, and the counters are then combined for decision making, the effect of noise can be eliminated with a finite arrival rate (current and power) of particles that matches the rate at which the bipolar detectors delete their memory.

The effect of currents of entangled particles is discussed and the corresponding current flows are computed in Section V. We show that if polarity switching errors are symmetric, then entanglement has no effect on the outcome of our analysis, both for the primary flows and the flows of interfering particles.

\section{THE MATHEMATICAL MODEL}

Consider a network of $m$ communicating nodes where some node $i$ communicates with another node $j$ by sending to it a Poisson flow of particles which may have negative or positive spin, of rate (intensity) $R_{i j}^{+}$and $R_{i j}^{-}$, respectively, so that $R_{i j}=R_{i j}^{+}+R_{i j}^{-}$. Some fraction $f_{i j, k}$ of the flow directed from $i$ to $j$ is diverted to node $k$ by errors induced by physical imperfections, causing unwanted interference at other receivers. The fraction of the flow that is not diverted and arrives at $j$ is: $F_{i j}=1-\sum_{k \neq j} f_{i j, k}$. Due to random effects in the course of transmission, some of these particles may change polarity from +1 to -1 with probability $Q_{i j}^{+}$, and from negative to positive spin with probability $Q_{i j}^{-}$. On top of this, "noise" particles of positive and negative spin also arrive to the receiver at rates $B_{j}, b_{j}$ respectively. As a result, the intensity of the total flow of particles with positive and negative spin arriving at node $j$ are $r_{i j}^{+}$and $r_{i j}^{-}$respectively:

$$
\begin{aligned}
r_{i j}^{+}= & F_{i j}\left[R_{i j}^{+}\left(1-Q_{i j}^{+}\right)+R_{i j}^{-} Q_{i j}^{-}\right]+B_{j} \\
& +\sum_{l=1, k=1 ; l \neq i, k \neq j}^{m, m} f_{l k, j}\left[R_{l k}^{+}\left(1-Q_{l j}^{+}\right)+R_{l k}^{-} Q_{l j}^{-}\right], \\
r_{i j}^{-}= & F_{i j}\left[R_{i j}^{-}\left(1-Q_{i j}^{-}\right)+R_{i j}^{+} Q_{i j}^{+}\right]+b_{j} \\
& +\sum_{l=1, k=1 ; l \neq i, k \neq j}^{m . m} f_{l k, j}\left[R_{l k}^{-}\left(1-Q_{l j}^{-}\right)+R_{l k}^{+} Q_{l j}^{+}\right] .
\end{aligned}
$$

We can write this as the sum of the signal, plus the noise including the interference:

$$
\begin{gathered}
r_{i j}^{+}=R_{i j}^{+}+N_{i j}, \\
r_{i j}^{-}=R_{i j}^{-}+n_{i j},
\end{gathered}
$$

where $\left(r_{i j}^{+}, r_{i j}^{-}\right)$represents the total received signal at $j$ resulting from the transmitted signal $\left(R_{i j}^{+}, R_{i j}^{-}\right)$, and the additional effects totalling $\left(N_{i j}, n_{i j}\right)$, which include the interference and the noise $B_{j}, b_{j}$ :

$$
\begin{aligned}
N_{i j}= & -R_{i j}^{+}\left(1-F_{i j}\right)+F_{i j}\left[R_{i j}^{-} Q_{i j}^{-}-R_{i j}^{+} Q_{i j}^{+}\right]+B_{j} \\
& +\sum_{l=1, k=1, k \neq j}^{m, m} f_{l k, j}\left[R_{l k}^{+}\left(1-Q_{l j}^{+}\right)+R_{l k}^{-} Q_{l j}^{-}\right], \\
n_{i j}= & -R_{i j}^{-}\left(1-F_{i j}\right)+F_{i j}\left[R_{i j}^{+} Q_{i j}^{+}-R_{i j}^{-} Q_{i j}^{-}\right]+b_{j} \\
& +\sum_{l=1, k=1, k \neq j}^{m, m} f_{l k, j}\left[R_{l k}^{-}\left(1-Q_{l j}^{-}\right)+R_{l k}^{+} Q_{l j}^{+}\right] .
\end{aligned}
$$

Let the total noise rate be $\beta_{j}=B_{j}+b_{j}$, and the total interference be $I_{i j}=I_{i j}^{+}+I_{i j}^{-}$where:

$$
\begin{aligned}
I_{i j}^{+} & =\sum_{l=1, k=1, k \neq j}^{m, m} f_{l k, j}\left[R_{l k}^{+}\left(1-Q_{l j}^{+}\right)+R_{l k}^{-} Q_{l j}^{-}\right], \\
I_{i j}^{-} & =\sum_{l=1, k=1, k \neq j}^{m, m} f_{l k, j}\left[R_{l k}^{-}\left(1-Q_{l j}^{-}\right)+R_{l k}^{+} Q_{l j}^{+}\right] .
\end{aligned}
$$

In the special case where the source $i$ sends particles to $j$ at rate $\Lambda$, assuming all the particles sent initially have positive spin, the total noise plus interference terms are:

$$
\begin{aligned}
N_{i j}^{+} & =-\Lambda\left(1-F_{i j}\right)-\Lambda F_{i j} Q_{i j}^{+}+B_{j}+I_{i j}^{+}, \\
n_{i j}^{+} & =\Lambda F_{i j} Q_{i j}^{+}+b_{j}+I_{i j}^{-},
\end{aligned}
$$

so that under the assumption that the positive and negative flows of pure noise $B_{j}=b_{j}$ are of equal rate, and that the interference positive and negative spike trains are also of equal rate $I_{i j}^{+}=I_{i j}^{-}$, we have

$$
\begin{aligned}
& N_{i j}^{+}-n_{i j}^{+}=-\Lambda\left(1-F_{i j}\right)-2 \Lambda F_{i j} Q_{i j}^{+}, \\
& N_{i j}^{+}+n_{i j}^{+}=-\Lambda\left(1-F_{i j}\right)+\beta_{j}+I_{i j} .
\end{aligned}
$$

Similarly if the source $i$ sends particles to $j$ at rate $\Lambda$, assuming all the particles sent initially have negative spin, then the total noise plus interference terms are:

$$
\begin{aligned}
N_{i j}^{-} & =\Lambda F_{i j} Q_{i j}^{-}+B_{j}+I_{i j}^{+}, \\
n_{i j}^{-} & =-\Lambda\left(1-F_{i j}\right)-\Lambda F_{i j} Q_{i j}^{-}+b_{j}+I_{i j}^{-} .
\end{aligned}
$$

Assuming that the positive and negative flows of pure noise $B_{j}=b_{j}$ are of equal intensity, and that the flows of positive and negative interference particles are also of equal intensity $I_{i j}^{+}=I_{i j}^{-}$, we have

$$
\begin{aligned}
& N_{i j}^{-}-n_{i j}^{-}=\Lambda\left(1-F_{i j}\right)+2 \Lambda F_{i j} Q_{i j}^{-}, \\
& N_{i j}^{-}+n_{i j}^{-}=\Lambda\left(1-F_{i j}\right)+\beta_{j}+I_{i j} .
\end{aligned}
$$


Remark 1: Under the only condition that $Q_{i j}^{+}=Q_{i j}^{-}$we see that $N_{i j}^{+}-n_{i j}^{+}=n_{i j}^{-}-N_{i j}^{-}$. Furthermore if also $B_{j}=b_{j}$ and $I_{i j}^{+}=I_{i j}^{-}$, it follows that $N_{i j}^{+}=n_{i j}^{-}$and $n_{i j}^{+}=N_{i j}^{-}$. These conditions as a whole will be called the Symmetric System Conditions.

\section{ERROR PROBABILITY FOR A SINGLE SPIN DETECTOR}

Single particle spin detectors have been suggested to detect polarity when single particles are used to transfer binary data. Thus it is of interest to predict the probability of error in the detection of bipolar signals carried by single particles, and we first consider the case without entangled particles. The probability that a positive spin particle is sent by the source $i$ to $j$ is:

$$
S_{i j}=\frac{R_{i j}^{+}}{R_{i j}}
$$

and obviously $\left(1-S_{i j}\right)$ is the probability that the sent particle's spin is negative. Since $R_{i j}$ is the total rate at which the sender $i$ sends particles to $j$, the receiver will expect to receive them on average each $1 / F_{i j} R_{i j}$ time units. Thus the probability that an interfering particle, from noise or other sources, arrives at $j$ before the particle that was sent from $i$ can arrive is:

$$
s_{i j}=\frac{I_{i j}+b_{j}+B_{j}}{I_{i j}+F_{i j} R_{i j}+b_{j}+B_{j}},
$$

in which case the receiver will interpret the interfering particle as being the original sent particle. Notice that $s_{i j}$ does not depend on the value of the bipolar random variable $V_{i j}$ representing the spin of a particle sent from $i$ to $j$. Also let $v_{i j}$ represent the spin of a received particle.

Since the error probability for the simple detector is due either to an interfering particle of opposite polarity being received, or to a change in polarity of the particle that is sent:

$$
\begin{aligned}
E_{i j} & =P\left[v_{i j}=-1 \cap V_{i j}=1\right]+P\left[v_{i j}=1 \cap V_{i j}=-1\right], \\
& =P\left[v_{i j}=-1 \mid V_{i j}=1\right] S_{i j}+P\left[v_{i j}=1 \mid V_{i j}=-1\right]\left(1-S_{i j}\right),
\end{aligned}
$$

and

$$
\begin{aligned}
E_{i j}= & {\left[\frac{I_{i j}^{-}+b_{j}}{I_{i j}+\beta_{j}} s_{i j}+\left(1-s_{i j}\right) Q_{i j}^{+}\right] S_{i j} } \\
& +\left[\frac{I_{i j}^{+}+B_{j}}{I_{i j}+\beta_{j}} s_{i j}+\left(1-s_{i j}\right) Q_{i j}^{-}\right]\left(1-S_{i j}\right) .
\end{aligned}
$$

Result 1: Assuming both spin polarities are equally probable for the transmitted particles $S_{i j}=\frac{1}{2}$ and writing $Q_{i j}=Q_{i j}^{+}+Q_{i j}^{-}$we have:

$$
E_{i j}=\frac{1}{2} \frac{I_{i j}+\beta_{j}}{F_{i j} R_{i j}+I_{i j}+\beta_{j}}+\frac{1}{2}\left(1-s_{i j}\right) Q_{i j} .
$$

If the total rate at which the source sends particles is $\Lambda=R_{i j}$ then:

$$
E_{i j}=\frac{1}{2} \frac{I_{i j}+\beta_{j}}{\Lambda F_{i j}+I_{i j}+\beta_{j}}+\frac{1}{2}\left(1-s_{i j}\right) Q_{i j} .
$$

Result 2 (See Fig. 1): When all the sources have identical behaviour, and those particles that are diverted as interference head towards other destinations with equal probability, then $I_{i j}=\Lambda\left(1-F_{i j}\right)$ so that after some algebra we have:

$$
E_{i j}=\frac{1}{2}-\frac{1}{2} \frac{\Lambda F_{i j}\left(1-Q_{i j}\right)}{\Lambda+\beta_{j}} .
$$

Remark 2: We can readily see from (22) that increasing the rate $\Lambda$ at which the particles are sent, will reduce the error, with a lower bound given as follows:

$$
E_{i j}^{\min }=\frac{1}{2}\left[1-F_{i j}\left(1-Q_{i j}\right)\right] \leq E_{i j} .
$$

$E_{i j}^{\min }$ can only be attained for $\beta_{j}>0$ when $\Lambda \rightarrow+\infty$.

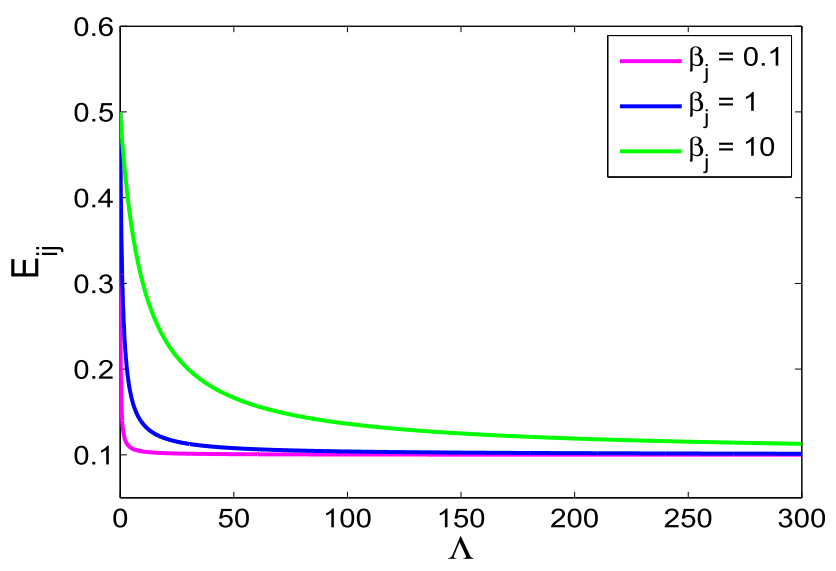

FIGURE 1. The error probability $E_{i j}$ plotted from (22) against $\Lambda$ for $F_{i j}\left(1-Q_{i j}\right)=0.8$ and three values of $\beta_{j}=0.1,1,10$.

\section{A. POWER AND ENERGY}

Remark 2 tells us that the error probability $E_{i j}$ can be reduced by increasing the rate at which particles are transmitted. However this will obviously come at some cost in terms of power and energy consumption. From the above discussion we see that increasing the rate at which particles are transmitted by a node can result in fewer errors. However, the increase in $\Lambda$ will result in greater energy consumption.

If the particles being considered are electrons, and a field of $V$ volts is used to move the electrons from the source to the destination node, then the amount of energy needed per electron will be $V$ Electron-Volts, or $E=V \times 1.602176565(35) \times 10^{-19}$ Joules. Hence the power consumption for each node to node communication will be directly proportional to $\Lambda$, i.e. $\Pi=\Lambda$. $E$.

Since the bit error probability per particle is $E_{i j}$, the rate of correctly received particles is computed via (22) as:

$$
D=\Lambda\left(1-E_{i j}\right)=\frac{\Lambda}{2}+\frac{\Lambda^{2} F_{i j}\left(1-Q_{i j}\right)}{2\left(\Lambda+\beta_{j}\right)},
$$

where we recall that $F_{i j}$ is the fraction of the flow sent from $i$ that does arrive at $j$. Notice also that the derivative of $D$ with 
respect to $\Lambda$ is:

$$
\frac{\partial D}{\partial \Lambda}=\frac{1}{2}\left[1+\frac{\Lambda^{2} F_{i j}\left(1-Q_{i j}\right)-1}{\left(\Lambda+\beta_{j}\right)^{2}}\right] .
$$

Since $F_{i j}$ should not be too small, and will be close to 1 for a properly designed system, and $\Lambda \gg 1$, we see that in the parameter ranges of interest $D$ will be an increasing function of $\Lambda$, which also suggests operating the system at higher power levels.

\section{NOISE REDUCTION VIA A BIPOLAR DETECTOR}

We will now suggest a detection scheme for the spin polarity which can lead to lower error probabilities based on the following principle:

- First, at each receiver $j$, the flows of arriving particles are channeled with equal probability into two distinct counters $H_{i j}(t), L_{i j}(t)$. Let $M_{i j}(t)=+1,-1$ if the receiver receives a particle at time $t$ that has positive or negative spin, respectively at time $t . M_{i j}(t)=0$ if no particle arrives at time $t$.

- The two counters are updated as follows:

- If $M_{i j}(t) \neq 0$ then either $H_{i j}(t)$ or $L_{i j}(t)$ is incremented with equal probability;

- If $H_{i j}(t)$ is incremented, then $H_{i j}\left(t^{+}\right)=\max [0$, $\left.H_{i j}(t)+M_{i j}(t)\right]$ where $t^{+}$denotes the instant right after $t$, i.e. $H_{i j}(t)$ is incremented by one whenever a positive spin particle arrives, and decremented by one (as long as its value is positive) if a negative spin particle arrives.

- Otherwise, $L_{i j}\left(t^{+}\right)=\max \left[0, H_{i j}-S_{i j}(t)\right]$, so that positive spin particles will decrement $L_{i j}(t)$ and increment $H_{i j}(t)$, proceeding in the opposite direction with the arrival of negative spin particles.

Note that neither counter can have a value which is negative, and that each one of them receives exactly one half of the flow arriving at the receiver $j$.

- Furthermore in order to limit their memory span, when a counter is positive it depletes its value at rate $\mu$ which represents "forgetfulness" with respect to older events. The detector $D_{i j}(t)$ then operates as follows:

- If $H_{i j}(t)>L_{i j}(t)$ then $D_{i j}(t)$ detects a +1 spin,

- If $L_{i j}(t)>H_{i j}(t)$, it detects a -1 spin,

- If $H_{i j}(t)=L_{i j}(t)$ then it detects either +1 or -1 at random with equal probability.

Therefore:

$$
\begin{aligned}
& P\left[D_{i j}(t)=1\right] \\
& \quad=P\left[H_{i j}(t)>L_{i j}(t)\right]+\frac{1}{2} P\left[H_{i j}(t)=L_{i j}(t)\right] .
\end{aligned}
$$

\section{A. ERROR ANALYSIS}

With Poisson arrivals for all particles, and exponentially distributed "forgetfulness", we can show that if $V_{i j}<1$ and $c_{i j}<1$ then:

$$
\begin{gathered}
\lim _{t \rightarrow \infty} P\left[H_{i j}(t)=k\right]=\left(1-C_{i j}\right) C_{i j}^{k}, \quad k=0,1, . . \\
\lim _{t \rightarrow \infty} P\left[L_{i j}(t)=k\right]=\left(1-c_{i j}\right) c_{i j}^{k}, \quad k=0,1, . .
\end{gathered}
$$

where:

$$
C_{i j}=\frac{R_{i j}^{+}+N_{i j}}{2 \mu+R_{i j}^{-}+n_{i j}}, \quad c_{i j}=\frac{R_{i j}^{-}+n_{i j}}{2 \mu+R_{i j}^{+}+N_{i j}},
$$

so that we can derive:

$$
\begin{aligned}
U_{i j}= & \lim _{t \rightarrow \infty} P\left[H_{i j}(t)>L_{i j}(t)\right]=\frac{C_{i j}\left(1-c_{i j}\right)}{1-C_{i j} c i j}, \\
u_{i j}= & \lim _{t \rightarrow \infty} P\left[H_{i j}(t)<L_{i j}(t)\right]=\frac{c_{i j}\left(1-C_{i j}\right)}{1-C_{i j} c i j}, \\
& \lim _{t \rightarrow} P\left[H_{i j}(t)=L_{i j}(t)\right]=\frac{1-C_{i j}-c_{i j}+C_{i j} c_{i j}}{1-C_{i j} c i j} .
\end{aligned}
$$

Assuming that we have equally likely polarities for $V_{i j}$, the probability of error for $D_{i j}(t)$ is:

$$
\begin{aligned}
e_{i j}(t)= & \frac{1}{2} P\left[D_{i j}(t)=-1 \cap V_{i j}=1\right] \\
& +\frac{1}{2} P\left[D_{i j}(t)=1 \cap V_{i j}=-1\right],
\end{aligned}
$$

and asymptotically we have:

$$
e_{i j}=\lim _{t \rightarrow \infty} e_{i j}(t)
$$

Now if the Symmetric System Conditions hold then $N^{+}=n^{-}$and $n^{+}=N^{-}$, and using Remark 1 we obtain.

Result 3: Under the Symmetric System Conditions, and assuming that all sent particles have positive spin:

$$
e_{i j}=\frac{1}{2}-\frac{1}{2} \frac{C_{i j}-c_{i j}}{1-C_{i j} c_{i j}}=\frac{1}{2}-\frac{\Lambda}{2 \mu} \frac{1}{2} F_{i j}\left(1-Q_{i j}\right) .
$$

Note that we must have $\Lambda<2 \mu$; but if we set $\Lambda$ as large as possible, i.e. $\Lambda \rightarrow 2 \mu$, then $e_{i j}$ is minimised:

$$
e_{i j}^{\min }=\frac{1}{2}\left[1-F_{i j}\right]+F_{i j} Q_{i j} \leq e_{i j} .
$$

Proof: We can write:

$$
\begin{aligned}
& \frac{C^{+}-c^{+}}{1-C^{+} c^{+}} \\
& =\frac{\Lambda^{2}+\left(N^{+}\right)^{2}+2 \Lambda N^{+}+2 \Lambda \mu+2 \mu N^{+}-2 \mu n^{+}-\left(n^{+}\right)^{2}}{4 \mu^{2}+2 \mu\left(N^{+} n^{+}\right)+n^{+} N^{+} 2 \mu \Lambda-\Lambda n^{+}-n^{+} N^{+}}, \\
& \left.=\frac{\Lambda}{2 \mu} \frac{\Lambda+N^{+}+n^{+}+2 \mu+\left(N^{+}-n^{+}\right) \frac{\Lambda+N^{+}+n^{+}+2 \mu}{\Lambda}}{2 \mu+N^{+}+n^{+}+\Lambda}\right],
\end{aligned}
$$

resulting in:

$$
\begin{aligned}
\frac{C^{+}-c^{+}}{1-C^{+} c^{+}} & =\frac{\Lambda}{2 \mu}\left[1+\frac{N^{+}-n^{+}}{\Lambda}\right], \\
& =\frac{\Lambda}{2 \mu}\left[1-\left(1-F_{i j}\right)-2 F_{i j} Q_{i j}^{+}\right] \\
& =\frac{\Lambda}{2 \mu} F_{i j}\left(1-2 Q_{i j}^{+}\right) .
\end{aligned}
$$

Now because the Symmetric System Conditions imply that $Q_{i j}=2 Q_{i j}^{+}$the proof is complete. 
Note that $e_{i j}^{\min }$ is the smallest possible value of the error that we may expect since it includes just the effect of the fraction of particles that do not arrive at the receiver, plus those that do arrive but which switch polarity.

Remark 5: We note that $e_{i j}$ does not depend at all on the "pure noise" term $\beta_{j}$. Thus the bipolar detector eliminates the noise term from error probability. Furthermore if we set $\beta_{j}=0$ in $E_{i j}$ of (22), we obtain $e_{i j}$ when $\Lambda=2 \mu$. Obviously the bipolar detector allows us to eliminate the noise effect $\beta_{j}$ from the error term, without requiring infinite power, i.e. at the power value $\Pi_{b}^{*}=4 \alpha \mu^{2}$.

Remark 6: With the bipolar detector, the system power need not exceed $\Pi=\Lambda V$ Electron-Volts, since at this level the detection error probability is zero.

\section{THE EFFECT OF ENTANGLED PARTICLE PAIRS}

Suppose that within each flow that a source $i$ sends to $j$, there are also two flows of positive and negative spin entangled particles of intensities $\lambda_{i j}^{+}, \lambda_{i j}^{-}$, where these intensities are equal so that $\lambda_{i j} \equiv \lambda_{i j}^{+}=\lambda_{i j}^{-}$. As with other particles, some of these may indeed find their way to $j$ with probability $F_{i j}$ while others may be diverted to some other channel with probability $f_{i j, k}$. As before, the probabilities $Q_{i j}^{+}$and $Q_{i j}^{-}$ represent the spin switching effect of the fields present in the channel $(i, j)$, and we denote by $r_{i j}^{2+}$ and $r_{i j}^{e-}$ the rate at which positive and negative spin particles are received from channel $(i, j)$ at $j$ in the presence of entanglement. Then because the companion entangled particles are assumed to switch spin in unison and in opposite direction:

- If, with probability $\left(F_{i j}\right)^{2}$ both entangled particles indeed go from $i$ to $j$, then

- The probability that a positive spin particle switches to negative, inducing its "partner" to switch to positive, is $Q_{i j}^{+}\left(1-Q_{i j}^{-}\right)$, resulting in no change in the flows of entangled particles from $i$ to $j$.

- While the probability that a negative spin particle switches to positive, with its positive companion switching the other way under this effect is $Q_{i j}^{-}\left(1-Q_{i j}^{+}\right)$again with no change in the flows of entangled particles,

- If both are induced to switch by the field in channel $(i, j)$ with probability $Q_{i j}^{+} \cdot Q_{i j}^{-}$, we will assume that the result is indeterminate and the switch may or may not occur, and in both cases there will be no change in the number and flow of entangled particles.

- If the particle pairs go into distinct channels with probability $F_{i j} \cdot\left(1-F_{i j}\right)$ then depending on whether the positive or negative spin particle that remains in the channel, as far as this particular channel $(i, j)$ is concerned they will behave singly; however they will create correlations with other channels.

- If the particle pairs, rather than entering channel $(i, j)$ go together into the channel $(i, k)$ with probability $\left(f_{i j, k}\right)^{2}$, then as above, the resulting flow is impervious to the switching effects of the fields in the channel.
Thus the equations (2), (3) with the inclusion of the effect of entangled particles become:

$$
\begin{aligned}
r_{i j}^{e+}= & F_{i j}\left[\left(R_{i j}^{+}-\lambda_{i j}\right)\left(1-Q_{i j}^{+}\right)+\left(R_{i j}^{-}-\lambda_{i j}\right) Q_{i j}^{-}\right] \\
& +F_{i j}\left(1-F_{i j}\right) \lambda_{i j}\left[Q_{i j}^{-}+1-Q_{i j}^{+}\right]+\left(F_{i j}\right)^{2} \lambda_{i j}+B_{j} \\
& +\sum_{\substack{l=1, k=1 \\
l \neq i, k \neq j}}^{m, m} f_{l k, j}\left[\left(R_{l k}^{+}-\lambda_{l k}\right)\left(1-Q_{l j}^{+}\right)+\left(R_{l k}^{-}-\lambda_{l k}\right) Q_{l j}^{-}\right] \\
& +\sum_{\substack{l=1, k=1 \\
l \neq i, k \neq j}}^{m, m} \lambda_{l k}\left[\left(f_{l k, j}\right)^{2}+f_{l k, j}\left(1-f_{l k, j}\right)\left(1-Q_{i j}^{+}+Q_{i j}^{-}\right)\right],
\end{aligned}
$$

$$
\begin{aligned}
r_{i j}^{e-}= & \left.F_{i j}\left(R_{i j}^{-}-\lambda_{i j}\right)\left(1-Q_{i j}^{-}\right)+\left(R_{i j}^{+}-\lambda_{i j}\right) Q_{i j}^{+}\right] \\
& +F_{i j}\left(1-F_{i j}\right) \lambda_{i j}\left[Q_{i j}^{+}+1-Q_{i j}^{-}\right]+\left(F_{i j}\right)^{2} \lambda_{i j}+b_{j} \\
& +\sum_{\substack{l=1, k=1 \\
l \neq i, k \neq j}}^{m, m} f_{l k, j}\left[\left(R_{l k}^{-}-\lambda_{l k}\right)\left(1-Q_{l j}^{-}\right)+\left(R_{l k}^{+}-\lambda_{l k}\right) Q_{l j}^{+}\right] \\
& +\sum_{\substack{l=1, k=1 \\
l \neq i, k \neq j}}^{m, m} \lambda_{l k}\left[\left(f_{l k, j}\right)^{2}+f_{l k, j}\left(1-f_{l k, j}\right)\left(1-Q_{l j}^{-}+Q_{l j}^{+}\right)\right],
\end{aligned}
$$

yielding:

$$
\begin{aligned}
r_{i j}^{e+}= & F_{i j}\left[R_{i j}^{+}\left(1-Q_{i j}^{+}\right)+R_{i j}^{-} Q_{i j}^{-}\right]+\lambda_{i j}\left(F_{i j}\right)^{2}\left(Q_{i j}^{+}-Q_{i j}^{-}\right) \\
& +\sum_{\substack{l=1, k=1 \\
l \neq i, k \neq j}}^{m, m} f_{l k, j}\left[R_{l k}^{+}\left(1-Q_{l j}^{+}\right)+R_{l k}^{-} Q_{l j}^{-}\right] \\
& +\sum_{\substack{l=1, k=1 \\
l \neq i, k \neq j}}^{m, m} \lambda_{l k}\left(f_{l k, j}\right)^{2}\left(Q_{l j}^{+}-Q_{l j}^{-}\right)+B_{j}, \\
r_{i j}^{e-}= & F_{i j}\left[R_{i j}^{-}\left(1-Q_{i j}^{-}\right)+R_{i j}^{+} Q_{i j}^{+}\right]+\lambda_{i j}\left(F_{i j}\right)^{2}\left(Q_{i j}^{-}-Q_{i j}^{+}\right) \\
& +\sum_{\substack{l=1, k=1 \\
l \neq i, k \neq j}}^{m, m} f_{l k, j}\left[R_{l k}^{-}\left(1-Q_{l j}^{-}\right)+R_{l k}^{+} Q_{l j}^{+}\right] \\
& +\sum_{\substack{l=1, k=1 \\
l \neq i, k \neq j}}^{m, m} \lambda_{l k}\left(f_{l k, j}\right)^{2}\left(Q_{l j}^{-}-Q_{l j}^{+}\right)+b_{j} .
\end{aligned}
$$

Remark 7: If $Q_{i j}^{+}=Q_{i j}^{-}$for all $(i, j)$ then all the $r_{i j}^{e+}=r_{i j}^{+}$ and $r_{i j}^{e-}=r_{i j}^{-}$term by term, and all the previous results that were derived without considering the effect of the flows of entangled particles, are identical when we also have entangled particles.

\section{CONCLUSION}

This paper has focused on the analysis of error probabilities for nano-communications between a set of interfering of $M$ nodes which may interfere with each other as they attempt to communicate via particles that have bipolar spins. We have 
assumed Poisson flows of particles being transmitted and received, and have included the effects of polarity switches, the interference between communicating pairs, and noise.

When all the error and noise effects are symmetric, we have derived the error probability for a simple detector, and the suggested a novel bipolar detector that eliminates the effects of noise at finite power as long as noise is symmetric. Power and energy considerations have also been introduced. We have also considered the specific effect of entangled particles and shown that they do not affect the overall results as long as spin polarity switches are symmetric.

Future work will focus on asymmetric systems, i.e. systems where the fields which may be viewed as noise result in asymmetric error probabilities. Also, we should consider multi-hop communications where a succession of nodes are traversed, either with a detection at each node and a transmission of a new flow of particles, or when the same particles are routed through multiple nodes, but with additional error effects at each stage.

\section{REFERENCES}

[1] E. Gelenbe and R. R. Muntz, "Probabilistic models of computer systemsPart I (exact results)," Acta Inf., vol. 7, no. 1, pp. 35-60, 1976.

[2] E. Gelenbe, "Probabilistic models of computer systems. Part II: Diffusion approximations, waiting times and batch arrivals," Acta Inf., vol. 12, no. 4, pp. 285-303, Nov. 1979.

[3] B. E. Kane, "A silicon-based nuclear spin quantum computer," Nature, vol. 393, pp. 133-137, May 1998.

[4] E. Gelenbe, "The first decade of G-networks," Eur. J. Oper. Res., vol. 126, no. 2, pp. 231-232, 2000.

[5] B. T. Jonker et al., "Robust electrical spin injection into a semiconductor heterostructure," Phys. Rev. B, vol. 62, no. 12, p. 8180, 2000.

[6] S. A. Wolf et al., "Spintronics: A spin-based electronics vision for the future," Science, vol. 294, no. 5546, pp. 1488-1495, 2001.

[7] J. A. Gupta, R. Knobel, N. Samarth, and D. D. Awschalom, "Ultrafast manipulation of electron spin coherence," Science, vol. 292, no. 5526, pp. 2458-2461, 2001.

[8] E. Uysal-Bıyıkog̃lu, B. Prabhakar, and A. El Gamal, "Energy-efficient packet transmission over a wireless link," IEEE/ACM Trans. Netw., vol. 10, no. 4, pp. 487-499, Aug. 2002.

[9] J. Medhi, Stochastic Models in Queueing Theory, 2nd ed. London, U.K.: Academic, 2003.

[10] F. Baccelli, E. Gelenbe, and B. Plateau, "An end-to-end approach to the resequencing problem," J. ACM, vol. 31, no. 3, pp. 474-485, Jul. 1984

[11] X. Jiang et al., "Optical detection of hot-electron spin injection into GaAs from a magnetic tunnel transistor source," Phys. Rev. Lett., vol. 90, p. 256603, Jun. 2003.

[12] P. Sharma, "How to create a spin current," Science, vol. 307, no. 5709, pp. 531-533, 2005.

[13] A. Goldsmith, Wireless Communications. Cambridge, U.K.: Cambridge Univ. Press, 2005.

[14] V. Percec et al., "Steric communication of chiral information observed in dendronized polyacetylenes," J. Amer. Chem. Soc., vol. 128, no. 50, pp. 16365-16372, 2006.

[15] J. Clayden, "Molecular devices: Communicating chirality," Nature Chem., vol. 3, pp. 842-843, Oct. 2011.

[16] X. Lou et al., "Electrical detection of spin transport in lateral ferromagnet-semiconductor devices," Nature Phys., vol. 3, pp. 197-202, Feb. 2007.
[17] C. L. Degen, M. Poggio, H. J. Mamin, and D. Rugar, "Role of spin noise in the detection of nanoscale ensembles of nuclear spins," Phys. Rev. Lett., vol. 99 , no. 25 , p. 250601 , Dec. 2007

[18] E. Gelenbe and G. Loukas, "A self-aware approach to denial of service defence," Comput. Netw., vol. 51, no. 5, pp. 1299-1314, Apr. 2007.

[19] Gartner, Inc. (2007). Gartner Estimates ICT Industry Accounts for $2 \%$ of Global $\mathrm{CO}_{2}$ Emissions. [Online]. Available: http:// www.gartner.com/newsroom/id/503867

[20] The Climate Group. SMART 2020: Enabling the Low Carbon Economy in the Information Age. [Online]. Available: http://www.smart2020.org/_assets/files/03_Smart2020Report_lo_res.pdf, accessed Jun. 2008.

[21] N. Dimakis, A. Filippoupolitis, and E. Gelenbe, "Distributed building evacuation simulator for smart emergency management," Comput. J., vol. 53, no. 9, pp. 1384-1400, 2010.

[22] E. Gelenbe and S. Timotheou, "Random neural networks with synchronized interactions," Neural Comput., vol. 20, no. 9, pp. 2308-2324, 2008.

[23] E. Gelenbe and C. Morfopoulou, "A framework for energy-aware routing in packet networks," Comput. J., vol. 54, no. 6, pp. 850-859, Jun. 2011.

[24] E. Gelenbe, D. Gesbert, D. Gesbert, D. Gündüz, H. Külah, and E. Uysal-Bıyıkog̃lu, "Energy harvesting communication networks: Optimization and demonstration (the E-CROPS project)," in Proc. 24th Tyrrhenian Int. Workshop Digit. Commun.-Green ICT (TIWDC), Genoa, Italy, Sep. 2013, pp. 1-6.

[25] E. Gelenbe and D. Gündüz, "Optimum power level for communications with interference," in Proc. 24th Tyrrhenian Int. Workshop Digital Commun.-Green ICT (TIWDC), Genoa, Italy, Sep. 2013, pp. 1-6.

[26] H. Yuan et al., "Zeeman-type spin splitting controlled by an electric field," Nature Phys., vol. 9, pp. 563-569, Jul. 2013.

[27] M. Kuscu, A. Kiraz, and O. B. Akan, "Fluorescent molecules as transceiver nanoantennas: The first practical and high-rate information transfer over a nanoscale communication channel based on FRET," Sci. Rep., vol. 5, Jan. 2015, Art. ID 7831.

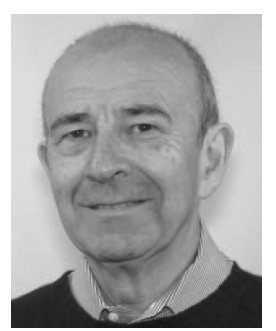

EROL GELENBE (F'86) received the $\mathrm{Ph} . \mathrm{D}$. degrees from the University of Liège, Belgium, the Università di Roma II, Italy, and Boğaziçi University, Istanbul. He founded the System Performance Evaluation Groups at IRIA, and in French universities, he was the Chair of the Electronics and Communication Engineering Department with Duke University, created the School of Electrical Engineering and Computer Science with the University of Central Florida, founded the team that designed the commercial queuing network analysis package, and developed the flexible manufacturing system simulator, the SYCOMORE multiprocessor switch, the XANTHOS fiber optics local area network, and the cognitive packet network routing algorithm. He also invented mathematical models, such as G-networks and the random neural network. He is currently the Dennis Gabor Professor and Head of Intelligent Systems and Networks with the Department of Electrical and Electronic Engineering, Imperial College London, where he is involved in energy savings, system security, and quality of service. He is a fellow of the French National Academy of Technologies, the Science Academies of Hungary, Poland and Turkey, the Royal Academy of Sciences, the Arts and Letters of Belgium, the Academia Europaea, and the Association for Computing Machinery (ACM). He received international prizes include the In Memoriam Dennis Gabor Award of the Hungarian Academy of Sciences, the Oliver Lodge Medal of the IET in London, the ACM-SIGMETRICS Life-Time Achievement Award, the Grand Prix France Télécom (currently Orange) of the French Academy of Sciences, the IFIP Silver Core Award, and the Parlar Foundation Science Award of Turkey. 